\title{
A Closer Look at Co-Rumination: Gender, Coping, Peer Functioning and Internalizing/Externalizing Problems
}

\author{
Tanya L. Tompkins \\ Linfield College \\ Ashlee R. Hockett \\ Linfield College \\ Nadia Abraibesh \\ Linfield College \\ Jody L. Witt \\ Linfield College
}

Follow this and additional works at: https://digitalcommons.linfield.edu/psycfac_pubs

Part of the Social Psychology Commons

\section{DigitalCommons@Linfield Citation}

Tompkins, Tanya L.; Hockett, Ashlee R.; Abraibesh, Nadia; and Witt, Jody L., "A Closer Look at CoRumination: Gender, Coping, Peer Functioning and Internalizing/Externalizing Problems" (2011). Faculty Publications. Accepted Version. Submission 6.

https://digitalcommons.linfield.edu/psycfac_pubs/6

This Accepted Version is protected by copyright and/or related rights. It is brought to you for free via open access, courtesy of DigitalCommons@Linfield, with permission from the rights-holder(s). Your use of this Accepted Version must comply with the Terms of Use for material posted in DigitalCommons@Linfield, or with other stated terms (such as a Creative Commons license) indicated in the record and/or on the work itself. For more information, or if you have questions about permitted uses, please contact digitalcommons@linfield.edu. 
A Closer Look at Co-Rumination: Gender, Coping, Peer Functioning and Internalizing/Externalizing Problems

Tanya L. Tompkins

Ashlee R. Hockett

Nadia Abraibesh

Jody L. Witt

Linfield College

Correspondence concerning this article should be addressed to Tanya L. Tompkins, Department of Psychology, Linfield College, 900 SE Baker Street, A570, McMinnville, OR 97128.

E-mail: tatompki@linfield.edu

Phone: 503-883-2684

Fax: 503-883-2669 


\begin{abstract}
Co-rumination, defined as repetitive, problem-focused talk explains higher levels of friendship quality in youth (Rose, 2002) and increased levels of anxiety/depression in females. Middle adolescents $(N=146)$ participated in a study of co-rumination, individual coping, externalizing/internalizing problems, and peer functioning. Consistent with past research, girls reported higher levels of co-rumination and internalizing symptoms. Co-rumination was also positively correlated with self-reports, but not teacher reports, of anxiety/depression and aggressive behavior. Both self-reported number of friends and teacher-rated social acceptance were negatively associated with co-rumination. Co-rumination partially accounted for the significant indirect effect of gender on internalizing symptoms. Additionally, co-rumination was associated with internalizing and externalizing symptoms but not individual coping efforts. Finally, co-rumination accounted for a unique amount of variance in internalizing symptoms, controlling for externalizing problems and secondary control coping. Theoretical implications and the importance of including broad domains of adjustment and peer functioning in future investigations of co-rumination are discussed.
\end{abstract}

Keywords: Co-rumination, Gender, Coping, Friendships, Internalizing Symptoms, Externalizing Problems 
A Closer Look at Co-Rumination: Gender, Coping, Peer Functioning and Internalizing/Externalizing Problems

During adolescence, friends surpass parents in their role as primary providers of social support (Furman \& Buhrmester, 1992). It has long been recognized that friendships contribute in key ways to youth's psychosocial functioning (Sullivan, 1953; Hartup, 1996). While most of this work has focused on the protective aspects of close friendships (see Rubin, Bukowski, \& Parker, 1998 for a review), there have been hints in the literature that some support processes are associated with maladjustment. For example, Gore and colleagues (1993) found that interpersonal caring orientation and involvement was associated with depression. Additionally, La Greca and Harrison (2005) found that while positive friendship qualities predicted lower social anxiety among adolescents studied, these did not protect them against feelings of depression. More recently, Schmidt and Bagwell (2007) found that for girls, while help and security functioned as protective factors in the presence of peer victimization, closeness increased depressive symptoms. For boys, the opposite effects emerged. However, not until Rose (2002) coined the term "co-rumination" did this notion, that aspects of peer support might have costs as well as benefits, gain traction and begin to receive systematic attention and study.

Co-rumination is defined as excessive discussion of problems within a close relationship and is characterized by mutual encouragement of problem-focused talk, rehashing problems, speculating about the problem in terms of causes and consequences, and dwelling on negative feelings (Rose, 2002). This integrative construct bridges two relatively independent literatures (friendship, coping) and explains contradictory findings. By late adolescence, girls are two to three times more likely to experience depression than boys (Nolen-Hoeksema \& Girgus, 1994). Given that girls tend to self-disclose in their same-sex friendships more than boys (McNelles \& 
Connolly, 1999) and enjoy closer friendships (Camarena, Sarigiani, \& Peterson, 1990), which are thought to protect against internalizing problems, it is surprising that girls remain more vulnerable to depression and anxiety relative to boys. Co-rumination may be one mechanism that explains these paradoxical findings.

\section{Co-Rumination: Explanation and Recent Findings}

Co-rumination is similar to self-disclosure, in that it involves sharing inner thoughts and feelings which may provide a means of building intimacy and gaining support in relationships (Rose, 2002); it shares with rumination, a strong, perseverative, and negative focus, which has been found to be associated with depression and impaired problem solving (Rose, 2002; NolenHoeksema, Wisco \& Lyubomirsky, 2008), and thus, may also come with a cost. Although defined as lying at the juncture of self-disclosure and rumination, co-rumination is distinct. Its negative, excessive, and repetitive focus extends beyond normative self-disclosure. Unlike rumination, an individual, cognitive/emotional process, co-rumination is a social process that may be reinforced by a partner (Rose, 2002).

Recent evidence has supported the notion of co-rumination as a social process that is associated with adjustment trade-offs. In her original study, Rose (2002) found that corumination was associated with internalizing symptoms, girls co-ruminated more than boys (and increasingly so with age), and co-rumination partially mediated the relationship between gender and anxiety/depression. As predicted, co-rumination was also associated with more positive friendship qualities, especially for boys.

In order to understand prospective relationships, Rose, Carlson, and Waller (2007) followed third-, fifth-, seventh- and ninth-graders over a 6-month period to determine whether co-rumination preceded changes in adjustment across time. In girls, there were transactional 
associations among co-rumination, friendship quality and emotional adjustment such that corumination predicted increasing anxiety and depression, but also increasing friendship quality over time. Positive friendship quality, depression, and anxiety (only for those in high quality friendships) also predicted increasing co-rumination across time. For boys, co-rumination was only associated with increasing friendship quality. The bi-directional nature of findings for girls suggests that the positive effects of co-rumination may perpetuate a vicious cycle of increased closeness with friends but higher levels of depression and anxiety as well. While these recent studies by Rose and colleagues suggest that co-rumination may be a depressogenic mechanism that partially explains the emergence of gender differences in depression, others have failed to find prospective increases in depressive symptoms among girls (Starr \& Davila, 2009).

Depression and Anxiety. Starr and Davila (2009) extended findings to demonstrate distinct relationships between social anxiety and depression in their relationship to corumination. Not unexpectedly, given its associations with low levels of self-disclosure (Cuming \& Rapee, 2010) and fewer and lower quality friendships (see Kingery, Erdley, Marshall, Whitaker, \& Reuter, 2010 for a review), they found that (when controlling for depression) social anxiety was negatively associated with co-rumination. However, in Rose and colleagues' (2007) investigation, anxiety (assessed with a measure primarily tapping worry and somatic aspects of anxiety, not social anxiety) demonstrated similar relationships with co-rumination as depression. Although alternative hierarchical models have been recently proposed (e.g., Lahey, Applegate, Waldman, Loft, Hankin, \& Rick, 2004) that not only differentiate anxiety and depression but various aspects of anxiety as well (e.g., fears vs. general anxiety and/or social anxiety that overlaps with depression), the current measure of internalizing symptoms does not adequately assess fears, separation anxiety and obsessions/compulsions which appear to constitute a separate 
anxiety factor, nor does it tap social anxiety which may be uniquely associated with corumination. In light of this and the fact that in her original work Rose employed a combined measure of anxiety/depression we also will examine the relationship between co-rumination and a combined measure of anxiety/depressive symptoms.

Developmental Context. Theoretical and empirical work suggests that as adolescents become more autonomous from parents, their friendships become increasingly intimate and offer an important source of social support (De Goede, Branje, \& Meeusy, 2009; Furman \& Buhrmester, 1992; Sullivan, 1953). There is also evidence that stress and coping responses vary across development in terms of the types of stressors adolescents experience as stressful, the competencies and behaviors adolescents can bring to bear to cope with stress, and the flexibility with which they can draw on their coping repertoire. For example, in a recent longitudinal study Seiffge-Krenke, Aunola and Nurmi (2009) found increases in the use of active and internal coping styles across early and late adolescence, with girls using higher levels of active coping, like seeking social support, relative to boys. Thus, in our sample of middle adolescents, it may be that strategies, such as co-rumination which share an approach-based focus, will be frequently used, especially by young women.

Prior studies of co-rumination and self-disclosure have supported the idea that gender differences in these social processes strengthen across adolescence (Rose, 2002; Rose et al., 2007; Rose \& Rudolph, 2006). However, in the sole published study of college students, gender differences were generally weaker than in studies involving younger samples (e.g., $d=1.27$ for adolescents in Rose et al., 2007 who were a combined group of $7^{\text {th }}$ and $9^{\text {th }}$ graders vs. $d=.69$ for college students in Calmes \& Roberts, 2008 sample). Thus, in the current study we seek to replicate and extend this work to an older adolescent sample expecting a significant relationship 
between co-rumination and internalizing problems, still sizable gender differences in corumination and evidence that co-rumination serves as a partial mediator of gender differences in emotional adjustment.

\section{Co-rumination and Externalizing Problems}

Although a growing number of studies have examined the association between corumination and internalizing problems, to our knowledge, no studies have investigated corumination's relationship with externalizing difficulties. This is surprising given the documented association between rumination and aggression/anger. Limited research has identified anger rumination as distinct from sadness rumination in predicting aggressive and depressed outcomes among both high-risk adolescents (Peled \& Moretti, 2007) and a normative sample of young adults (Peled \& Moretti, 2010). Co-rumination may be associated with externalizing problems given the conceptual overlap with anger rumination. The possibility that co-rumination is associated with externalizing, in addition to internalizing problems, will be explored in the current study.

\section{Co-rumination and Peer Functioning}

While co-rumination has generally been associated with positive friendship quality, the relationships between this construct and other facets of the peer relationship (e.g., social acceptance, network size) have been understudied. Only one study has examined other aspects

of peer functioning, finding that while cross-sectional relationships generally supported the view of a direct relationship between co-rumination and positive peer outcomes, prospective relationships were suggestive of deleterious peer outcomes with baseline co-rumination predicting a decrease in the number of friends one year later (Starr \& Davila, 2009). It may be that while the self-disclosing nature of co-rumination enhances intimacy and closeness within a 
dyad, the repetitive and negative focus instigates other friendship processes (e.g., selection, influence) that diminish the peer network size leaving the friends who are most similar in terms of internalizing problems and/or the tendency to co-ruminate. Dynamics of the individual or relationship may influence the tendency for contagion and amplification of negative affect or the withdrawal and rejection of a co-ruminating friend. Given the lack of prior research attention to the issue, the strength and direction of correlations among co-rumination and other aspects of peer relationships will be explored.

\section{Coping, Gender, Co-rumination and Psychological Symptoms}

Increasingly large numbers of children and adolescents exhibit severe emotional and behavioral problems (Achenbach, Dumenci, \& Rescorla, 2002). Theoretically, prevailing etiological models recognize the importance of stress and coping in the development and maintenance of emotional and behavioral difficulties in youth. Practically, both prevention and intervention efforts that seek to enhance the capacity of youth and families to cope with stress are vital (Compas, Connor-Smith, Saltzman, Thomsen, \& Wadsworth, 2001).

Although a burgeoning body of work examining the nature and correlates of coping in children and adolescents has accrued (see Compas et al., 2001 and Clarke, 2006 for reviews), until recently, the study of coping in youth has been complicated by a lack of clarity and consensus in defining and measuring the construct (Connor-Smith, Compas, Wadsworth, Thomsen, \& Saltzman, 2000; Compas et al., 2001). Prominent theoretical models (Ayers, Sandler, West, \& Roosa, 1996; Walker, Smith, Garber, \& Van Slyke, 1997) of coping highlight key dimensions of voluntary coping, including coping efforts that: are enacted to exert control over the stressor or one's reaction to it; represent a child/adolescent's attempts to adapt to the stressful experience; and involve disengaging from the stressor and/or one's emotional response 
to the stressor (Compas et al. 2001). The current project adopted the five-factor Responses to Stress Model (Connor-Smith et al., 2000).

The Responses to Stress Questionnaire (RSQ) was developed to tap: primary control coping (problem solving, emotional regulation, and emotional expression), secondary control coping (positive thinking, cognitive restructuring, acceptance, and distraction), disengagement coping (avoidance, denial, and wishful thinking), involuntary engagement (rumination, intrusive thoughts, emotional and physiological arousal, and impulsive action), and involuntary disengagement (emotional numbing, cognitive interference, inaction, and escape). Strengths of this measure include: volitional coping items that minimize overlap with symptom distress; multiple versions of the measure which prompt the participant to report on coping with a particular type of stressor (e.g., family conflict, interpersonal stress); and proportion scores to control for base rate differences in endorsement of strategies (Connor-Smith et al., 2000).

Studies in the coping literature that have specifically employed the Responses to Stress Model (Connor-Smith et al., 2000; DeCarlo Santiago \& Wadsworth, 2009; Wadsworth \& Compas, 2002) suggest that coping responses are an important correlate of psychological adjustment (Compas et al., 2001). In general, studies suggest that engaging in primary and secondary control coping are associated with more favorable youth outcomes (e.g., fewer internalizing/externalizing problems, higher social competence), whereas disengagement strategies are associated with less favorable outcomes. These general trends, however, tend to be qualified by the nature of the stressor, the specific types of coping used, and whether these associations are measured concurrently or prospectively (Compas et al., 2001).

Whether co-rumination is associated with individual coping, beyond rumination and other involuntary engagement responses, and the degree to which it predicts unique variance in 
outcome are open questions. It is similar to support seeking which cuts across primary control coping. There are limited data (see Starr \& Davila, 2009 for a discussion of the role of romantic experiences) and speculation that suggest that co-rumination may be a way to cope with stress. If seeking social support and expressing emotions is a common individual coping strategy it seems reasonable that co-rumination which involves rehashing, speculating and dwelling may also be a typical way to cope with stress. Theoretically, co-rumination is thought to be associated with depression because it involves dwelling on negative affect and prevents friends from engaging in activities that could distract from the problem. Similarly, the passive, perseverative, and speculative focus on details, causes, and consequences may also be associated with anxiety given its future-oriented focus. Finally, if the co-ruminative thoughts and feelings center on frustration or anger, the same perserverative and speculative processes that contribute to depression/anxiety may also be associated with externalizing behaviors given overlap with anger rumination. The current project represents the first attempt to understand how corumination is associated with individual coping efforts and the degree to which it predicts overlapping and unique variance in internalizing and externalizing problems.

\section{The Current Study}

Rose and colleagues $(2002 ; 2007)$ have urged the continued study of this integrative construct, which lies at the juncture of self-disclosure and rumination, given its' promise to enhance our understanding of the developmental course of self-disclosure, friendship quality, coping responses, and psychological well-being. Unfortunately, her call has been neglected. In summary, we seek to replicate prior studies using a middle adolescent sample, expecting that: adolescent girls will report higher levels of co-rumination relative to boys, co-rumination will concurrently be associated with higher levels of internalizing symptoms, and co-rumination will 
partially explain gender differences in internalizing problems. To extend knowledge of corumination in new directions, we explored several additional questions: (1) is co-rumination associated with externalizing behaviors? (2) is co-rumination associated with peer acceptance and peer network size? (3) to what extent is co-rumination associated with individual coping efforts? (4) does co-rumination predict unique variance in outcomes?

\section{Method}

\section{Participants}

The participants were 146 adolescents (101 girls; 69\%) enrolled in a public high school in an exurban school district in the Pacific Northwest where $36 \%$ of the students qualified for free or reduced-price lunch. Adolescents ranged in age from 14 to $19(M=16.84, S D=1.09) ; 11 \%$ were $9^{\text {th }}$ graders, $14 \%$ were $10^{\text {th }}$ graders, $34 \%$ were $11^{\text {th }}$ graders, and $41 \%$ were $12^{\text {th }}$ graders. Reflecting the ethnic composition of the surrounding region, the sample was $75 \%$ Caucasian, 11\% Hispanic/Latino, 5\% Asian-American and 8\% mixed/other. Data were missing from 15 students due to absenteeism or incomplete responses. Additionally, data were not available for all 146 participating students for all measures, primarily due to unforeseen disruption during data collection with two classrooms involving approximately 30 students. To maximize power across analyses we included participants that were missing data from no more than one study measure and listwise deletion was used to deal with missing data.

\section{Procedure}

Teachers from a broad range of classes (e.g., social sciences, math, science, music, physical education) were identified by the school administrator as teaching multiple classes across grade levels. These teachers distributed consent forms to adolescents, who brought them home for parents' consideration along with a letter encouraging parents to return the consent. 
Consent forms were returned for approximately $50 \%$ of the adolescents, $94 \%$ for whom parents provided permission. All adolescents, whose parents provided consent, assented to take part in the study. Study procedures were reviewed and approved by the Institutional Review Board (IRB) and all study data, except endorsement of suicidal thoughts/feelings which was shared with school counselors, were kept confidential.

Although participants completed additional measures as a part of a study on family stress and coping, only those relevant to the current study are described below. Trained research assistants administered the study questionnaires during group assessment sessions that lasted about 1 hour. Students provided the name of a teacher who knew them well enough to complete two brief measures about their behavior in the classroom setting. These teachers were then contacted and asked to complete the Teacher Report Form (TRF) and the Self-Perception Profile for Adolescents (SPPA) - Teacher Rating Scale (TRS). Participating teacher's received \$10 (with \$5 per student rating for more than two students). Teachers completed measures for a total of $93(64 \%)$ of the students.

\section{Measures}

Co-Rumination. The Co-Rumination Questionnaire (CQ; Rose, 2002), a 27-item selfreport inventory, assessed the extent to which youth typically co-ruminate with same-sex friends including: frequently discussing problems, mutual encouragement of problem talk, rehashing problems, speculating about problems, and dwelling on negative affect. Participants rated how well each statement described their interactions with a best or close friend using a 5-point Likerttype scale, ranging from 1 (not at all true) to 5 (really true). Sample items include: "When we talk about a problem that one of us has we will talk about every part of the problem over and over"; "When we talk about a problem that one of us has we talk for a long time about how upset 
it has made one of us with the problem"; and "When we talk about a problem that one of us has we talk a lot about all of the different bad things that might happen because of the problem." Corumination scores were the average rating across the 27 items, with higher scores reflecting higher levels of co-rumination. Cronbach's alpha for the current sample was .96. The measure has been used in child, adolescent (Rose, 2002: Rose et al. 2007; Starr \& Davila, 2009) and college (Calmes \& Roberts, 2008) samples with similarly high internal consistency ( $\alpha=.90$ to 97).

Psychological Adjustment. To assess emotional and behavior problems, adolescents completed the Youth Self-Report Form (YSR; Achenbach, \& Rescorla, 2001), a wellstandardized, reliable, and multidimensional measure of youth behavioral and emotional adjustment. Teachers completed the TRF (Achenbach, \& Rescorla, 2001), a parallel version of the YSR. Respondents rate 112 items describing problem behaviors experienced over the past 6 months on a 3-point scale ranging from 0 (not true) to 2 (very true or often true). The measure yields a total problem score, two second-order broadband categories of internalizing and externalizing behavior problems, and eight empirically derived narrow-band scales addressing a broad spectrum of emotional and behavioral symptoms. The eight narrow-band scales are labeled withdrawn, somatic complaints, anxious/depressed, social problems, thought problems, attention problems, delinquent behavior, and aggressive behavior. The current study focused on two subscales of the YSR/TRF: Anxious/Depressed (A/D) and Aggressive Behavior (AGG). These two scales are representative of internalizing and externalizing problems in adolescents and more closely match measures of psychopathology used in prior research relative to broadband internalizing and externalizing dimensions (e.g., Rose, 2002; Wadsworth \& Berger, 2006). Internal consistency, as measured by Cronbach's alpha, was acceptable: 
Anxiety/Depression $(\mathrm{A} / \mathrm{D})-\mathrm{YSR}(13$ items $)=.86$; TRF $(16$ items $)=.85$, Aggression $(\mathrm{AGG})-$ YSR $(17$ items $)=.83 ; \operatorname{TRF}(20$ items $)=.84$. Although one of two studies (Starr \& Davila, 2009) that has evaluated anxiety and depression as unique constructs found distinct relationships with co-rumination, measurement issues precluded our examination of the two symptom patterns separately.

\section{Peer Relationships}

Although data regarding friendship quality were not available, two indicators of peer relationships were assessed. First, youth were asked to report the total number of friends in their current peer network ("How many friends would you consider to be close friends? These are friends who you see more than once a week. These are friends who you spend time with and enjoy doing things with.").

Second, teacher reports of youth competence were gathered with the TRS version of the SPPA (Harter, 1988). Only the Social Acceptance (2 items) scale was of interest to the current study. Teachers responded to a forced-choice format, whereby they first chose which of two statements about the adolescent was most consistent with their impressions of him/her (e.g., Social Acceptance - "This individual does not have a lot of friends" or "This individual does have a lot of friends") and then indicated whether their choice was sort of true or really true. Items were converted to a 4-point scoring system, with higher scores reflecting greater perceived competence. Harter (1988) reported adequate reliability for the various scales across different samples; the social acceptance domain $(\alpha=.88)$ showed good internal consistency.

Coping. Adolescents completed the Responses to Stress Questionnaire (Family Conflict - Version) (RSQ; Connor-Smith et al., 2000), which assesses both effortful coping and involuntary stress reactions in response to family conflict. ${ }^{3}$ Participants rated 60 items on a 4- 
point Likert-type scale ranging from 1 (never) to 4 (a lot) yielding 19 scales that comprise five broad factors (first three representing voluntary coping strategies; last two representing involuntary stress responses): Primary Control Engagement $(\alpha=.80)$ includes items measuring problem solving $(\alpha=.59)$, emotional expression $(\alpha=.75)$, and emotional regulation $(\alpha=.46)$. The Secondary Control Engagement $(\alpha=.80)$ factor is comprised of items measuring strategies such as acceptance $(\alpha=.51)$, cognitive restructuring $(\alpha=.52)$, distraction $(\alpha=.44)$, and positive thinking $(\alpha=.69)$. Disengagement coping $(\alpha=.86)$ consists of strategies such as avoidance $(\alpha$ $=.80)$, denial $(\alpha=.43)$, and wishful thinking $(\alpha=.72)$. Involuntary Engagement $(\alpha=.92)$ includes rumination $(\alpha=.76)$, intrusive thoughts $(\alpha=.72)$, physiological arousal $(\alpha=.79)$, emotional arousal $(\alpha=.70)$, and impulsive action $(\alpha=.80)$. Involuntary Disengagement responses $(\alpha=.85)$ include emotional numbing $(\alpha=.43)$, cognitive interference $(\alpha=.72)$, inaction $(\alpha=.60)$, and escape $(\alpha=.62)$. The psychometric properties of the RSQ have been well established across samples of varying ages and SES levels (Connor-Smith et al., 2000). Coping scores represent the sum of the items on each of the 19 scales and the sum of each subscale loading on each of the five factors. The sum of all 60 items represents the total coping responses scores. The current alphas (noted above) are consistent with past studies and tend to be lower for subscales given the small number of items comprising each scale and higher for broad voluntary coping factors (i.e., primary control, secondary control, disengagement coping) and involuntary coping responses (i.e., involuntary engagement, involuntary disengagement).

\section{Results}

\section{Preliminary Analyses}

The measures of adjustment were significantly positively skewed: YSR-A/D (skew = 1.08$, standard error $=.20 ; z=5.40, p<.05)$, YSR-AGG $($ skew $=1.04$, standard error $=.20 ; z=$ 
$5.20, p<.05)$, TRF-A/D (skew $=2.43$, standard error $=.25 ; z=9.72, p<.05)$, TRF-AGG $($ skew $=2.90$, standard error $=.25 ; z=11.60, p<.05)$. This is consistent with expectations in that few student participants would be expected to be at high risk for clinical levels of symptoms. Measures of adjustment were normalized considerably by square root and logarithmic transformations. All analyses were run with both transformed and non-transformed variables. Results were similar with no changes in outcomes of inferential tests. Given that the distributions of these variables closely match expected population distributions and that correlation-based analyses are robust to skew we present analyses with non-transformed data. All other variable distributions were normally distributed.

\section{Gender Differences}

To examine gender differences in co-rumination and various indices of emotional/behavioral problems a series of independent samples $t$-tests were conducted. The results are summarized with descriptive statistics presented separately by gender in Table 1. As expected, adolescent girls reported higher levels of co-rumination and internalizing problems, relative to boys. These gender differences, however, were not found for any indicators of externalizing problems, nor for teacher reports of any emotional/behavioral problems.

\section{Relations between Co-Rumination, Adjustment, and Peer Relations}

Pearson correlations computed between co-rumination and adjustment variables are presented in Table 2 (along with correlations among all other coping variables). As expected, co-rumination was significantly and positively correlated with self-reported internalizing symptoms; however, it was not significantly associated with teacher-reported anxious/depressed symptoms. Co-rumination was also significantly and directly associated with self-reported externalizing problems, but again not with teacher reports of similar behaviors. Teacher-rated 
social acceptance was negatively related to co-rumination as well as both teacher and youth reports of internalizing (not externalizing) problems. Number of friends was not significantly associated with internalizing or externalizing behaviors, but was negatively associated with corumination. Providing some evidence of convergent validity across reporters, self-reported number of friends was positively associated with teacher reports of social acceptance.

\section{Co-Rumination as a Mediator between Gender and Adjustment}

To test whether co-rumination partially mediated gender differences in internalizing symptoms we used a bootstrapping method (Preacher \& Hayes, 2004) that evaluates the significance of the indirect effects.

As shown in Figure 1, the direct effects of gender on anxious/depressed symptoms and co-rumination were both significant with girls reporting higher levels of anxiety/depression and co-rumination relative to boys. The proposed mediator, co-rumination, significantly predicted internalizing symptoms while controlling for gender, with higher levels of co-rumination predicting more problematic adjustment. Finally, the effect of gender on internalizing symptoms while controlling for the effects of co-rumination, although not as strong, was still significant. The bootstrapped estimate of the indirect effect of gender on internalizing symptoms, through co-rumination, was statistically significant, as its $95 \%$ confidence interval (from .011 to .278) did not contain a zero. Thus, being female predicted more co-rumination and co-rumination, in turn, was associated with higher levels of anxious/depressed symptoms.

\section{Relations between Co-Rumination, Individual Coping, and Adjustment}

Girls reported more coping responses (see Table 1) and in line with Connor-Smith et al.'s (2000) recommendation, proportion scores on the RSQ were used in all analyses to address base rate differences in endorsement of coping responses. Consistent with past research, 
disengagement coping and involuntary coping responses were positively associated with both internalizing and externalizing symptoms, although relationships with externalizing problems were generally weaker (see Table 2). In fact, the only significant relationship between coping and teacher reports of adjustment was found between involuntary engagement and anxiety/depression. In contrast, both primary and secondary control coping were associated with fewer internalizing symptoms according to both self- and teacher-report. However, primary and secondary control coping were associated with fewer externalizing problems (although these relationships reached statistical significance only for self-reported difficulties). Interestingly, corumination, which is conceptualized as a social coping process, was not associated with individual coping efforts that controlled for base rate differences in coping responding. It was, however, positively associated with total coping responses (see Table 2).

To understand the unique relationships among coping strategies, co-rumination and symptoms, two separate hierarchical regression analyses were performed with either YSRanxious/depressed or YSR-aggressive behavior as outcome variables. In terms of predictor variables, on the first step, aggressive behavior was controlled in analyses predicting anxiety/depression and anxiety/depression was controlled in analyses predicting aggressive behavior given that the two were strongly related. Next, three coping variables (primary control, secondary control and involuntary engagement) were entered. To reduce the number of predictors given the small sample size and multicollinearity problems associated with the use of a set of proportion scores, the three variables most similar to the engagement/approach dimension of co-rumination were selected. Last, co-rumination was entered to determine if this social process predicted unique variance in adjustment above and beyond comorbid problems and individual coping strategies/responses. In addition to comorbid problems and secondary 
control coping, co-rumination appears to account for a unique amount of variance in internalizing symptoms (see Table 3). Neither coping nor co-rumination were independent predictors of externalizing problems once the effects of comorbid internalizing symptoms were accounted for.

\section{Discussion}

Our study contributes to a small body of research suggesting that co-rumination may be one gender-linked relationship process that helps explain gender differences in internalizing problems (Calmes \& Roberts, 2008; Rose \& Rudolph, 2006; Rose, 2002; Rose et al. 2007). We extend this research in important new directions by examining associations with externalizing problems, aspects of peer relations previously neglected, and individual coping skills within a sample of middle adolescents.

\section{Gender, Co-Rumination, and Peer/Emotional Adjustment}

Internalizing symptoms. Consistent with past research (Calmes \& Roberts, 2008; Rose, 2002; Rose et al., 2007), girls reported engaging in more co-rumination than boys. We also replicated past findings of gender differences in reports of depression and anxiety, with girls reporting significantly higher levels of internalizing symptoms (Rescorla et al., 2007). The mediation analyses replicated prior results with younger (Rose, 2002; Rose 2007) and older (Calmes \& Roberts, 2008) samples suggesting that co-rumination may account for gender differences in depression/anxiety. Specifically, girls reported co-ruminating more frequently within their close friendships. Girls who co-ruminated more also reported higher levels of selfreported depression/anxiety. Given that co-rumination has been previously found to partially mediate the relationship between gender and internalizing problems across three separate age and 
research groups, it may be one plausible mechanism to explain increased risk for these types of problems among girls and young women. Comparing gender differences in co-rumination across samples, it appears as if these differences intensify through early adolescence but begin to narrow shortly thereafter (e.g., $d=.67$ in the current sample vs. 1.27 in Rose's early adolescent sample and .69 in Calmes and Roberts' college sample). In light of emerging evidence suggesting that co-rumination predicts increasing depression across time (see Starr and Davila, 2009 for an exception), it is critical to investigate factors that explain the use of co-rumination amongst girls and potential risk factors associated with this unique coping mechanism.

One promising approach lies in adopting lab-based paradigms and bridging theory used in the study of rumination to understand distinct and common mechanisms across these two forms of repetitive thought. For example, Watkins (2008) recently elaborated a control theory framework for understanding repetitive thought (RT) whereby unconstructive outcomes of RT result from "...attention on the discrepancy between the desired goal and the actual situation, making the unresolved discrepancy more salient, perpetuating the unresolved issue, and exacerbating negative affect" (p. 191). Specifically, he highlighted the importance of three factors in accounting for consequences that arise in relation to repetitive thought: the valence of cognition (positive or negative), the intrapersonal and situational context of the individual engaged in RT (e.g., existing mood state, self beliefs), and the level of construal (concrete or abstract). The interpersonal context of the co-ruminating dyad adds another important dimension to consider. Whether and how the nature, process and outcomes of dyadic vs. individual repetitive thought differ is a ripe area for future inquiry. For example, does level of abstraction relating to a recent social stressor vary by gender and result in similar outcomes for both solitary and dyadic rumination? Do certain thought patterns such as self-blame, threat appraisal and/or 
low self-efficacy differentiate the negative effects that seem to accrue primarily to girls?

Studying rumination, in concert with co-rumination, promises to enhance our understanding of the ways in which thinking, action, interpersonal interaction, and emotional state interact.

Externalizing problems. The current study is the first to document a cross-sectional association between co-rumination and self-reported externalizing problems. Although there was a positive correlation between co-rumination and externalizing problems, gender differences in externalizing behaviors were not found. While most research on the conceptually similar process of rumination focuses on depression, there is a nascent literature examining anger in relationship to rumination among youth (Peled \& Moretti, 2007) and adults (Bushman, 2002; Rusting \& Nolen-Hoeksema, 1998). According to the amplification hypothesis, when repetitive thought has a negative focus (as it does in co-rumination), it worsens the pre-existing mood state and amplifies the reciprocal interplay between cognitions and mood (Ciesla \& Roberts, 2007). Evidence suggests that rumination exacerbates pre-existing anger (Rusting \& Nolen-Hoeksema, 1998), and increases the probability of an angry response to provocation (Bushman, Bonacci, Pedersen, Vasquez, \& Miller, 2005) in adults. In a clinical sample of adolescents, Peled and Moretti (2007) found that anger and sadness rumination were associated with emotional and behavioral problems in distinct ways. Furthermore, anger rumination predicted unique variance in anger, as well as relational and overt aggression, whereas sadness rumination only predicted depression. Whether co-rumination may similarly fuel an angry mood deserves further consideration and cannot adequately be answered on the basis of a cross-sectional association between co-rumination and externalizing problems. Future studies should examine whether the social nature of co-rumination serves to worsen emotion/behavior on the externalizing spectrum. 
Peer Relations. Mirroring results from studies of younger children, there was a significant association between teacher-rated social acceptance and both teacher and youth reports of internalizing problems (Klima \& Repetti, 2008). Self-reported number of friends was not significantly associated with internalizing or externalizing behavior, but was positively associated with teacher reports of social acceptance and negatively related to co-rumination. Highlighting the need to consider multiple components of youth's peer networks, the current results suggest that co-rumination may come at a cost in terms of social acceptance and peer network size. These results are consistent with Starr and Davila's (2009) finding of corumination being prospectively associated with fewer female friends across time. Additionally, Waller and Rose (2010) recently found that co-rumination was associated with adjustment tradeoffs in a sample of mothers and their adolescent children. In their study, not only was motheradolescent co-rumination related to positive relationship quality but it was also associated with greater mother/adolescent enmeshment and internalizing problems. Although not directly relevant to friendships and network size these results do suggest that there may be both interpersonal benefits and costs associated with co-rumination.

Unfortunately, friendship quality was not assessed, nor was friendship reciprocity. Thus, it is unclear whether there are both costs and benefits in the domain of peer functioning. Additionally, given the cross-sectional nature of the current data the possible direction of effects is unknown. For instance, it may be that the tendency to co-ruminate in one's closest friendship limits opportunities for expanding the peer network and increasing acceptance (either because of an intense focus on problem talk within a close friendship and/or because the tendency to coruminate might elicit burnout and social avoidance from others). Alternatively, it may be that a 
limited social network and poor acceptance increases social problems that provide topics for corumination.

Coping, Co-Rumination and Adjustment. This study is the first to examine the relationship between coping and co-rumination. Consistent with prior work (see Compas et al., 2001 for a review), disengagement coping strategies and involuntary coping responses were positively associated with both internalizing and externalizing symptoms, although relationships with externalizing problems and teacher reports of emotional adjustment were generally weaker. In contrast, and as supported by prior research, both primary and secondary control coping were associated in predicted directions with self- and teacher-reports of internalizing symptoms, but only with self-reports of externalizing problems.

Although no specific predictions regarding the relationship between co-rumination and individual coping variables were advanced, it was surprising that no significant bivariate relationships were found; particularly given the conceptual overlap with primary control coping and involuntary engagement. The one exception was a positive association between total coping responses and co-rumination. Some coping researchers have noted that endorsement of more coping strategies does not always signal more effective coping behavior (Steele, Cushing, Bender, \& Richards, 2008). Perhaps middle adolescents who adopt a wide range of coping strategies to cope with family conflict may also co-ruminate with friends about this and other sources of stress.

Results suggested that, in addition to externalizing symptoms and secondary control coping, co-rumination accounts for a small, but unique, amount of variance in internalizing symptoms. Neither coping, nor co-rumination predicted unique variance in externalizing problems once the effects of internalizing symptoms were accounted for. These results await 
replication but suggest that co-rumination is a unique social process that confers risk and is deserving of additional study.

\section{Limitations and Future Directions}

Despite several strengths, our study has some limitations. The analyses were conducted with an ethnically homogenous sample that included a small number of males. Additionally, the relatively high non-response rate necessarily limits generalizations that can be drawn about broader community samples of high school students. Second, as our sample was a community sample, future studies should examine coping processes, including co-rumination, within clinical samples to understand the extent to which they may buffer or exacerbate early symptoms. The assessment of adolescent's social acceptance solely through teacher ratings represents another limitation of the study. Because self- and/or peer-nominations were not obtained, the total number of friends identified may or may not include reciprocal friendships. Future longitudinal studies that incorporate sociometric assessments promise to enhance our understanding of whether co-ruminating youth have fewer reciprocal friends because they are less socially competent (which might suggest few friendship nominations from others, and provide social problems on which to co-ruminate with their limited network), or because they choose to have a small, close-knit friendship network of individuals (which might suggest they are selective in their reciprocal friendship nominations or that they have a small but highly reciprocal group of friends). Additionally, the study would have benefited from inclusion of a measure of friendship quality to replicate past findings of adjustment benefits in an older adolescent sample, while concurrently documenting adjustment costs in other peer domains. Future studies, particularly those that are longitudinal in nature and include measures across multiple domains of peer functioning, promise to enhance our understanding of the ways that co-rumination impacts peer 
relations more broadly. Including measures of multiple aspects of both peer functioning and emotional adjustment across time will provide an opportunity to deepen our understanding of the complex interplay between symptoms and social impairment.

The study would have been strengthened had we used a measure of coping that focused on peer-related stress. Given the focus of the larger study on family stress, to prevent participant burden, only the family stress version of the RSQ was used. Recent evidence underscores the importance of situational determinants of coping. For example, Seiffge-Krenke et al., 2009 found that situational variation explained between $50 \%$ and $69 \%$ of the variance in the three coping styles they examined. This and other research (Bowker, Bukowski, Hymel, \& Sippola, 2000; Griffith, Dubow, \& Ippolito, 2000) has suggested that youth use more active coping strategies in response to school- and peer-related sources of stress than situations involving family-related stress. Thus, it is likely that the relationships between co-rumination and coping might have been stronger had we used the social-stress version of the RSQ. Studying corumination and coping ratings in response to the same stressor would sharpen our understanding of the ways in which this dyadic process thwarts or enhances individual coping efforts and/or stress responses. Examining how this may vary across different types of stress (e.g., peer, family, financial) also promises to enhance our understanding of the ways in which interdependent and independent life stress may interact with co-rumination and individual coping efforts in predicting emotional adjustment. Employing sophisticated methods beyond simple self-report (e.g., lab-based generation of a stressor with self-reported coping and observed corumination; experience sampling) will help clarify how co-rumination fits into adolescents' coping repertoires and whether this unique social coping process is more likely to occur in response to certain sources of stress than others. 
Reliance on self-reported coping and co-rumination cannot exclude the influence of gender-specific response styles especially given the failure to detect significant and reliable associations between coping (including co-rumination) and teacher reports of emotional adjustment. However, past studies have supported similar patterns of gender differences based on peer- or self-reported coping (Causey \& Dubow, 1992) and have shown moderate associations between parent- and self-reports of coping. Additionally, the smaller sample size for analyses involving teacher data resulted in lower power; in some cases relationships were not drastically different (e.g., relationship between co-rumination and anxiety/depression was .26 for self-report and .19 for teacher report). Given that co-rumination is a dyadic process, future research will benefit from the inclusion of self and peer reports of co-rumination in the relationship and the modeling of actor and partner effects on outcome. Furthermore, coding labbased and/or time-sampled observational data may prove useful in understanding how problem content, importance and salience influence the tendency to co-ruminate within friendships and whether certain aspects of the process moderate outcome.

In closing, this growing body of work also has important applied implications. Worry and concern about those at risk for internalizing outcomes is typically focused on the socially isolated and withdrawn (e.g., Laursen, Bukowski, Aunola, \& Nurmi, 2007). For this reason, those in friendships characterized by high levels of co-rumination may be overlooked, allowing them to continue engaging in a social process that may strengthen a relationship but leave them vulnerable to negative emotional outcomes and a diminished social network. Incorporating this information into existing prevention and treatment programs may substantially improve outcomes. 


\section{Acknowledgements}

This research was supported by a faculty development grant from Linfield College. The authors would like to thank Chipo Dendere, Mandy Hamilton, Annelise Nelson, Ashley Reichelt, Jennifer Schenk, and Jessica Wilson for their assistance with data collection and/or data entry. Portions of these results were presented at the 2007 annual meeting of the Society for Research on Child Development in Boston, MA. 


\section{References}

Achenbach, T. M., Dumenci, L., \& Rescorla, L. A. (2002). Ten-year comparisons of problems and competencies for national samples of youth: Self, parent, and teacher reports. Journal of Emotional and Behavioral Disorders, 10, 194-203.

Achenbach, T. M., \& Rescorla, L. A. (2001). Manual for ASEBA school-Age forms \& profiles. Burlington, VT: University of Vermont, Research Center for Children, Youth, \& Families.

Ayers, T. S., Sandler, I. N., West, S. G., \& Roosa, M. W. (1996). A dispositional and situational assessment of children's coping: Testing alternative models of coping. Journal of Personality, 64, 923-958.

Bowker, A., Bukowski, W.M., Hymel, S., \& Sippola, L.K. (2000). Coping with daily hassles in the peer group during early adolescence: Variations as a function of peer experience. Journal of Research on Adolescence, 10, 211-243.

Bushman, B. J. (2002). Does venting anger feed or extinguish the flame? Catharsis, rumination, distraction, anger, and aggressive responding. Personality and Social Psychology Bulletin, 28, 724-731.

Bushman, B. J., Bonacci, A. M., Pedersen, W. C., Vasquez, E. A., \& Miller, N. (2005). Chewing on it can chew you up: Effects of rumination on triggered displaced aggression. Journal of Personality and Social Psychology, 88, 969-983.

Calmes, C.A., \& Roberts J.E. (2008). Rumination in interpersonal relationships: Does corumination explain gender differences in emotional distress and relationship satisfaction among college students? Cognitive Therapy and Research, 32, 577-590.

Camarena, P. M., Sarigiani, P. A., \& Peterson, A. C. (1990). Gender-specific pathways 
to intimacy in early adolescence. Journal of Youth and Adolescence, 19, 19-32.

Causey, D. L, \& Dubow, E. F. (1992). The development of a self-report coping measure for elementary school children. Journal of Clinical Child Psychology, 21, 47-59.

Ciesla, J. A. \& Roberts, J. E. (2007). Rumination, negative cognition, and their interactive effects on depressed mood. Emotion, 7, 555-565.

Clarke, A. T. (2006). Coping with interpersonal stress and psychosocial health among children and adolescents: A meta-analysis. Journal of Youth and Adolescence, 35, 11-24.

Compas, B. E., Connor-Smith, J. K., Saltzman, H., Thomsen, A. H., \& Wadsworth, M. E. (2001). Coping with stress during childhood and adolescence: Problems, progress and potential in theory and research. Psychological Bulletin, 127, 87-127.

Connor-Smith, J. K., Compas, B. E., Wadsworth, M. E., Thomsen, A. H., \& Saltzman, H. (2000). Responses to stress in adolescence: Measurement of coping and involuntary stress responses. Journal of Consulting and Clinical Psychology, 68, 976-992.

Cuming, S., \& Rapee, R. M. (2010). Social anxiety and self-protective communication style in close relationships. Behaviour, Research, and Therapy, 48, 87-96.

DeCarlo Santiago, C., \& Wadsworth, M.E. (2009). Coping with family conflict: What's helpful and what's not for low-income adolescents. Journal of Child and Family Studies, 18, 192-202.

De Goede, I. H. A., Branje, S. J. T., \& Meeusy, W. H. J. (2009). Developmental changes and gender differences in adolescents' perceptions of friendships. Journal of Adolescence, $32,1105-1123$.

Furman, W., \& Buhrmester, D. (1992). Age and sex differences in perceptions of networks of personal relationships. Child Development, 63, 103-115. 
Gore, S., Aseltine, R. H., \& Colten, M. E. (1993). Gender, social-relational involvement, and depression. Journal of Research on Adolescence, 3, 101-125.

Griffith, M.A., Dubow, E.F., \& Ippolito, M.F. (2000). Developmental and cross-situational differences in adolescents' coping strategies. Journal of Youth and Adolescence, 29, 183-204.

Harter, S. (1988). Manual for the self-perception profile for adolescents. Denver, CO: University of Denver.

Hartup, W. W. (1996). The company they keep: Friendships and their developmental significance. Child Development, 67, 1-13.

Kingery, J. N., Erdley, C. A., Marshall, K. C., Whitaker, K. G., \& Reuter, T. R. (2010). Peer experiences of anxious and socially withdrawn youth: An integrative review of the developmental and clinical literature. Clinical and Child Family Psychology Review, 13, 91-128.

Klima, T., \& Repetti, R. L. (2008). Children's peer relations and their psychological adjustment: Differences between close friendships and the larger peer group. Merrill-Palmer Quarterly, 54, 151-178.

La Greca, A. M. \& Harrison, H. M. (2005). Adolescent peer relations, friendships, and romantic relationships: Do they predict social anxiety and depression? Journal of Clinical Child and Adolescent Psychology, 34, 49-61.

Lahey, B. B., Applegate, B., Waldman, I. D., Loft, J. D., Hankin, B. L., \& Rick, J. (2004). The structure of child and adolescent psychopathology: Generating new hypotheses. Journal of Abnormal Psychology, 3, 358-385.

Laursen, B., Bukowski, W. M., Aunola, K., \& Nurmi, J. (2007). Friendship moderates 
prospective associations between social isolation and adjustment problems in young children. Child Development, 78, 395 - 1404.

McNelles, L. R., \& Connolly, J. A. (1999). Intimacy between adolescent friends: Age and gender differences in intimate affect and intimate behaviors. Journal of Research on Adolescence, 9, 143-159.

Nolen-Hoeksema, S., \& Girgus, J. S. (1994). The emergence of gender differences in depression during adolescence. Psychological Bulletin, 115, 424-443.

Nolen-Hoeksema, S., Wisco, B., \& Lyubomirsky, S. (2008). Rethinking rumination. Perspectives on Psychological Science, 3, 400-424.

Peled, M., \& Moretti, M. M. (2007). Rumination on anger and sadness in adolescence: Fueling of fury and deepening of despair. Journal of Clinical Child and Adolescent Psychology, 36, 66-75.

Peled, M., \& Moretti, M. M. (2010). Ruminating on rumination: Are rumination on anger and sadness differentially related to aggression and depressed mood? Journal of Psychopathology and Behavioral Assessment, 32, 108-117.

Preacher, K. J., \& Hayes, A. F. (2004). SPSS and SAS procedures for estimating indirect effects in simple mediation models. Behavior Research Methods, Instruments, \& Computers, 36, 717-731.

Rescorla, L., Achenbach, T., Ivanova, M. Y., Dumenci, L., Almqvist, F., Bilenberg, N., et al. (2007). Behavioral and emotional problems reported by parents of children ages 6 to 16 in 31 societies. Journal of Emotional and Behavioral Disorders, 15, 130-142.

Rose, A. J. (2002). Co-rumination in the friendships of girls and boys. Child Development, $73,1830-1843$. 
Rose, A. J., \& Rudolph, K. D. (2006). A review of sex differences in peer relationship processes: Potential trade-offs for the emotional and behavioral development of girls and boys. Psychological Bulletin, 132, 98-131.

Rose, A.J., Carlson, W., \& Waller, E.M. (2007). Prospective associations of corumination with friendship and emotional adjustment: Considering the socioemotional trade-offs of co-rumination. Developmental Psychology, 43, 1019-1031.

Rubin, K. H., Bukowski,W., \& Parker, J. G. (1998). Peer interactions, relationships, and groups. In W. Damon, \& N. Eisenberg (Eds.), Handbook of child psychology, Vol. 3: Social, emotional, and personality development, (pp. 619-700). New York: John Wiley \& Sons, Inc.

Rusting, C. L., \& Nolen-Hoeksema, S. (1998). Regulating responses to anger: Effects of rumination and distraction on angry mood. Journal of Personality and Social Psychology, $74,790-803$.

Schmidt, M. E., \& Bagwell, C. L. (2007). The protective role of friendships in overtly and relationally victimized boys and girls. Merrill-Palmer Quarterly, 53, 439-460.

Seiffge-Krenke, I., Aunola, K., \& Nurmi, J. (2009). Changes in stress perception and coping during adolescence: The role of situational and personal factors. Child Development, 80, 259-279.

Starr, L. R., \& Davila, J. (2009). Clarifying co-rumination: Associations with internalizing symptoms and romantic involvement among adolescent girls. Journal of Adolescence, 32, 19-37.

Steele, R. G., Cushing, C. C., Bender, J. A., \& Richards, M. M. (2008). Profiles and correlates 
of children's self-reported coping strategies using a cluster analytic approach. Journal of Child and Family Studies, 17, 140-153.

Sullivan, H. S. (1953). The interpersonal theory of psychiatry. New York: Norton.

Wadsworth, M. E., \& Berger, L. E. (2006). Adolescents coping with poverty-related family stress: Prospective predictors. Journal of Youth and Adolescence, 35, 57-70.

Wadsworth, M. E., \& Compas, B. E. (2002). Coping with family conflict and economic strain: The adolescent perspective. Journal of Research on Adolescence, 12, 243-274.

Walker, L. S., Smith, C. A., Garber, J., \& Van Slyke, D. A. (1997). Development and validation of the Pain Response Inventory for Children. Psychological Assessment, 9, $392-405$.

Waller, E.M., \& Rose, A.J. (2010). Adjustment trade-offs of co-rumination in mother-adolescent relationships. Journal of Adolescence, 33, 487-497.

Watkins, E. R. (2008). Constructive and unconstructive repetitive thought. Psychological Bulletin, 134, 163-206. 
Table 1

Descriptive Statistics and Gender Differences in Co-rumination, Coping and Adjustment

\begin{tabular}{lccccc}
\hline & Girls & Boys & & \\
Variables & $M(S D)$ & $M(S D)$ & $t$ test & $p$ value & Cohen's $d$ \\
\hline Co-rumination & $2.55(.79)$ & $2.07(.63)$ & $t(112)=3.00$ & .003 & .67 \\
RSQ - Total Responses & $126.37(26.73)$ & $116.77(25.39)$ & $t(142)=2.03$ & .044 & .37 \\
YSR-A/D & $7.00(5.25)$ & $3.81(3.41)$ & $t(122)=4.35^{\text {a }}$ & $<.001$ & .72 \\
YSR.-AGG & $6.91(4.55)$ & $7.92(5.78)$ & $t(66)=-1.02^{\text {a }}$ & .313 & .19 \\
TRF-A/D & $2.64(3.72)$ & $1.70(2.35)$ & $t(92)=1.24$ & .220 & .30 \\
TRF-AGG & $1.21(2.43)$ & $1.67(3.20)$ & $t(94)=-0.78$ & .435 & .17 \\
TRS - Social Acceptance & $3.12(.73)$ & $3.02(.79)$ & $t(89)=0.59$ & .554 & .13 \\
Number of Friends & $5.10(2.91)$ & $7.16(10.11)$ & $t(25)=-1.01^{\text {a }}$ & .324 & .28 \\
\hline
\end{tabular}

Note. RSQ = Responses to Stress Questionnaire - all scores used are proportion scores; YSR (Youth Self-Report)-A/D =

Anxious/Depressed; YSR-AGG = Aggressive Behavior; TRF (Teacher Report Form)-A/D = Anxious/Depressed; TRF-AGG = Aggressive Behavior; TRS - Harter's Teacher Rating Scale.

${ }^{a}$ Given a significant $F$ for Levene's test $t$ and $d f$ reported are for equal variances not assumed.

${ }^{\mathrm{b}}$ Two male participants who reported a large number of friends were removed as potential outliers in these and subsequent analyses.

Given that results were virtually unchanged their data were included. 


\section{Table 2}

Correlations Between Co-Rumination, Coping, and Adjustment

\begin{tabular}{|c|c|c|c|c|c|c|c|c|c|c|c|c|c|c|}
\hline & Variables & 1 & 2 & 3 & 4 & 5 & 6 & 7 & 8 & 9 & 10 & 11 & 12 & 13 \\
\hline 1 & Co-Rumination & -- & & & & & & & & & & & & \\
\hline 2 & RSQ-Primary & .03 & -- & & & & & & & & & & & \\
\hline 3 & RSQ - Second. & -.08 & $.33 * * *$ & -- & & & & & & & & & & \\
\hline 4 & RSQ - Diseng. & .04 & $-.65 * * *$ & $-.31 * * *$ & -- & & & & & & & & & \\
\hline 5 & RSQ - Inv. Eng. & .08 & $-.40 * * *$ & $-.73 * * *$ & -.02 & -- & & & & & & & & \\
\hline 6 & RSQ - Inv. Dis. & .01 & $-.65 * * *$ & $-.61 * * *$ & $.45^{* * *}$ & $.38 * * *$ & -- & & & & & & & \\
\hline 7 & RSQ - Total & $.29 * *$ & $-.37 * * *$ & $-.43 * * *$ & $.18^{*}$ & $.43 * * *$ & $.33 * * *$ & -- & & & & & & \\
\hline 8 & YSR - A/D & $.26 * *$ & $-.33 * * *$ & $-.47 * * *$ & $.29 * * *$ & $.45 * * *$ & $.31 * * *$ & $.56 * * *$ & -- & & & & & \\
\hline 9 & YSR - AGG & $.20 *$ & $-.28 * * *$ & $-.30 * * *$ & .16 & $.27 * * *$ & $.30 * * *$ & $.39 * * *$ & $.39 * * *$ & -- & & & & \\
\hline 10 & $\mathrm{TRF}-\mathrm{A} / \mathrm{D}$ & .19 & $-.25 *$ & $-.21 *$ & .15 & $.23 *$ & .20 & $.28 * *$ & $.42 * * *$ & .12 & -- & & & \\
\hline 11 & $\mathrm{TRF}-\mathrm{AGG}$ & .14 & .10 & .05 & -.12 & .04 & -.10 & .08 & .07 & $.24 *$ & $.33 * * *$ & -- & & \\
\hline 12 & TRS - Soc. Acc. & $-.27 *$ & $.23^{*}$ & .15 & -.19 & -.19 & -.10 & $-.23 *$ & $-.24 *$ & -.08 & $-.33 * *$ & -.12 & -- & \\
\hline 13 & No. of Friends & $.22 *$ & .09 & .05 & .00 & -.12 & -.05 & -.07 & -.01 & -.07 & -.12 & -.02 & $.27^{*}$ & -- \\
\hline
\end{tabular}


Table 2, continued

Note. Sample sizes across correlations vary from 70 to 144 . RSQ = Responses to Stress Questionnaire; RSQ-Second. = RSQ-Secondary Control; RSQ-Diseng. = RSQ-Disengagement Coping; RSQ-Inv. Eng. = RSQ-Involuntary Engagement; RSQ-Inv. Diseng. = RSQ-

Involuntary Disengagement; YSR (Youth Self-Report)-A/D = Anxious/Depressed; YSR-AGG = Aggressive Behavior; TRF (Teacher Report Form); TRS-Soc. Acc. $=$ Harter's Teacher Rating Scale - Social Acceptance.

$* p<.05$. ** $p<.01$. *** $p<.001$. 
Table 3

Hierarchical Regression Analysis Summary for Coping Variables and Co-Rumination Predicting Adolescent's Internalizing and

Externalizing Symptoms $(N=108)$

Variable

Step 1: Adjustment Problems

YSR - Anxious/Depressed

YSR - Aggressive Behavior

Step 2: Coping

RSQ - Primary Control

RSQ - Secondary Control

RSQ - Involuntary Engagement

\begin{tabular}{c}
$\begin{array}{c}\text { Internalizing Symptoms } \\
\text { (Anxious/Depressed) }\end{array}$ \\
\hline$B \quad R^{2} \quad \Delta R^{2}$
\end{tabular}

$.25 * * *$

---

$.32 * * *$

Externalizing Symptoms
(Aggressive Behavior)

$.25 * * *$

$.43 * * *$

---

$-.10$

Step 3:

Co-Rumination (CQ)

$.17^{*}$

$46 * * *$

$.02 *$

.10

Note. $\beta \mathrm{s}$ are standardized regression coefficients in the final model.

$* p<.05$. ** $p<.01$. *** $p<.001$. 


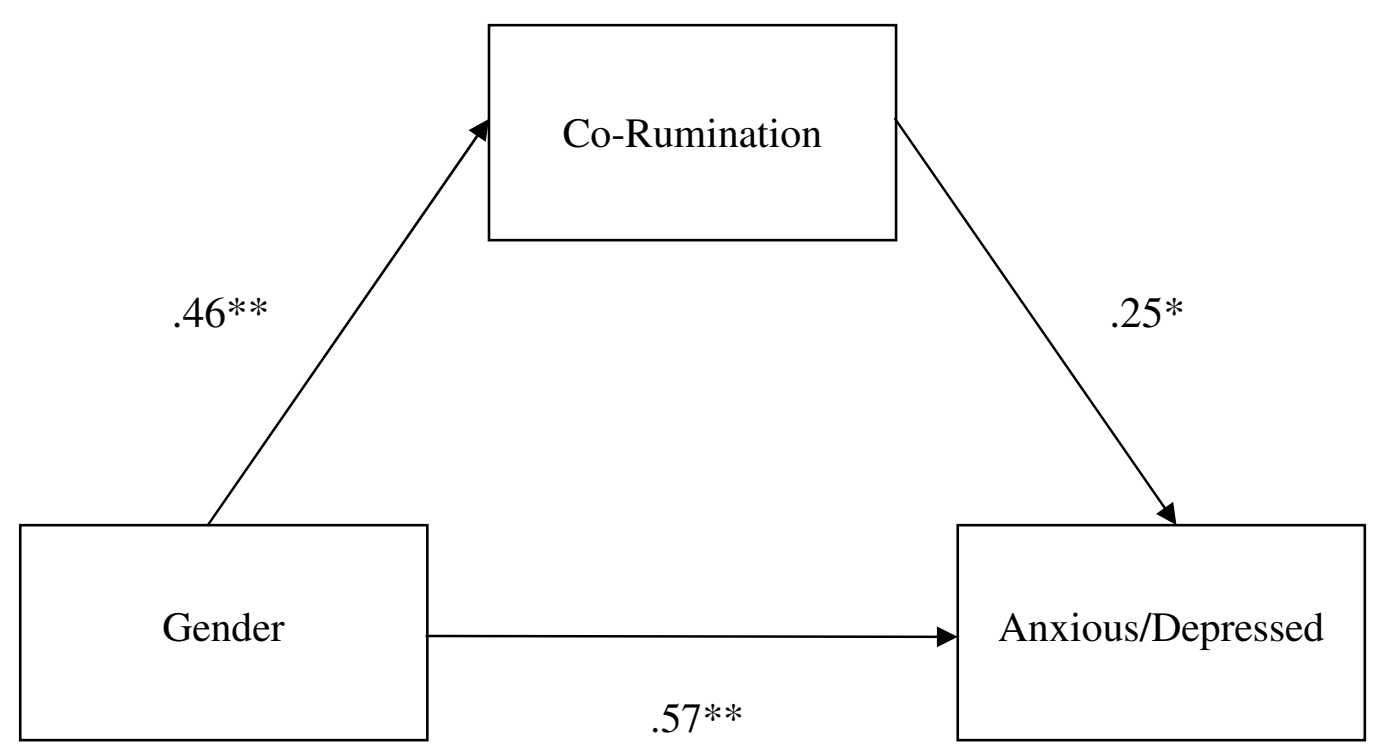

$\left(.45^{*}\right)$

Figure 1. Numbers in this figure are standardized $\beta$ s from a bootstrapping model testing the effects of co-rumination as a mediator. The number in parentheses represents the $\beta$ computed for gender predicted internalizing symptoms, while controlling for corumination. Boys were coded as 0 and girls were coded as 1 . $* p<.05 . * * p<.01$. 